\title{
Article \\ Safety and Efficacy of Weekly Paclitaxel and Cisplatin Chemotherapy for Ovarian Cancer Patients with Hypersensitivity to Carboplatin
}

\author{
Shinichi Tate*(D), Kyoko Nishikimi, Ayumu Matsuoka, Satoyo Otsuka and Makio Shozu \\ Department of Gynecology, Chiba University Hospital, 1-8-1 Inohana, Chuo-ku, Chiba 260-8670, Japan; \\ knishikimi@hospital.chiba-u.jp (K.N.); a-matsuoka@chiba-u.jp (A.M.); caxa5597@chiba-u.jp (S.O.); \\ shozu@faculty.chiba-u.jp (M.S.) \\ * Correspondence: state@faculty.chiba-u.jp; Tel.: +81-43-226-2121
}

Citation: Tate, S.; Nishikimi, K.; Matsuoka, A.; Otsuka, S.; Shozu, M. Safety and Efficacy of Weekly Paclitaxel and Cisplatin Chemotherapy for Ovarian Cancer Patients with Hypersensitivity to Carboplatin. Cancers 2021, 13, 640. https://doi.org/10.3390/ cancers 13040640

Academic Editor: Mandi Murph Received: 24 December 2020

Accepted: 29 January 2021

Published: 5 February 2021

Publisher's Note: MDPI stays neutral with regard to jurisdictional claims in published maps and institutional affiliations.

Simple Summary: This study was conducted to evaluate the safety and efficacy of weekly paclitaxel and cisplatin chemotherapy in patients with ovarian cancer who developed carboplatin hypersensitivity reaction. Eighty-six (86) patients who developed hypersensitivity reactions for carboplatin were treated with weekly paclitaxel and cisplatin chemotherapy, and $71(83 \%)$ of the 86 patients were able to receive treatment without hypersensitivity reaction to cisplatin. The severity of the hypersensitivity reaction for cisplatin observed in all 15 patients was below grade 2 , and there were no deaths due to hypersensitivity reaction to cisplatin. The majority of patients (55 patients, $64 \%$ ) completed the scheduled weekly paclitaxel and cisplatin chemotherapy, and only 9 patients $(10 \%)$ discontinued treatment due to hypersensitivity reaction within 6 cycles. Weekly paclitaxel and cisplatin chemotherapy were well-tolerated and effective for patients who developed carboplatin hypersensitivity reaction.

Abstract: Background: This study aimed to evaluate the safety and efficacy of weekly paclitaxel and cisplatin chemotherapy (wTP) in patients with ovarian cancer who developed carboplatin hypersensitivity reaction (HSR). Methods: We retrospectively investigated 86 patients with ovarian, fallopian tube, and peritoneal carcinoma who developed carboplatin HSR during previous chemotherapy (carboplatin and paclitaxel) at our institution between 2011 and 2019. After premedication was administered, paclitaxel was administered over $1 \mathrm{~h}$, followed by cisplatin over $1 \mathrm{~h}$ (paclitaxel $80 \mathrm{mg} / \mathrm{m}^{2}$; cisplatin $25 \mathrm{mg} / \mathrm{m}^{2} ; 1,8,15$ day $/ 4$ weeks). We investigated the incidence of patients who successfully received wTP for at least one cycle, treatments compliance, progression-free survival (PFS), and overall survival (OS). Results: The median number of wTP administration cycles was 4 (Interquartile Range IQR, 3-7), 71 patients (83\%) successfully received wTP, and 15 patients (17\%) developed cisplatin HSR. The efficacy of treatment was as follows: $55(64 \%)$ patients completed the scheduled wTP, $9(10 \%)$ patients discontinued due to HSR to cisplatin within 6 cycles, $1(1 \%)$ patient discontinued due to renal toxicity (grade 2) at the 6th cycle, and $21(24 \%)$ patients discontinued due to progressive disease within 6 cycles. The median PFS and OS after administration of wTP were 10.9 months (95\% CI: 7.7-17.7) and 25.9 months (95\% CI: 19.0-50.2), respectively. Conclusions: wTP was safe and well-tolerated in patients who developed carboplatin HSR.

Keywords: carboplatin; cisplatin; hypersensitivity reaction; ovarian cancer; platinum-sensitive disease; recurrence

\section{Introduction}

The standard therapy for platinum-sensitive recurrent ovarian cancer is a platinumbased chemotherapy plus bevacizumab (Bev) [1] or poly (adenosine diphosphate [ADP]ribose) polymerase (PARP) inhibitor maintenance [2] in case of response to platinum, and it is now influenced by the first-line treatment (use of Bev or PARPi). PARP inhibitors 
have been reported to be more effective in survival than Bev in patients with breast cancer susceptibility (BRCA) mutations in a network meta-analysis [3]. Moreover, a recent study has reported that carboplatin plus pegylated liposomal doxorubicin is the best chemotherapy regimen in combination with Bev for platinum-sensitive recurrent ovarian cancer [4] Furthermore, the Desktop III trial revealed the impact of secondary cytoreduction in platinum-sensitive recurrent ovarian cancer [5], while the Gynecologic Oncology Group (GOG) 0213 trial did not [6].

However, one of the problems in the treatment of platinum-sensitive recurrent ovarian cancers is the occurrence of carboplatin hypersensitivity reaction (HSR) [7]. The frequency of carboplatin hypersensitivity reaction increases with repeated exposure [7]. After such a reaction has occurred, it is difficult to use platinum compounds, despite the patients having platinum-sensitive disease. The absence of platinum administration due to carboplatin hypersensitivity reaction consequently makes it difficult to determine the platinum sensitivity of each patient; therefore, they cannot benefit from poly (adenosine diphosphate [ADP]-ribose) polymerase (PARP) inhibitors [2], for which platinum sensitivity is a clinical biomarker [8].

Alternative treatments for patients who developed carboplatin hypersensitivity reactions include changing to non-platinum drugs, more intense premedication, changing to other platinum drugs [9], or using a desensitization therapy for carboplatin [10,11]. Desensitization therapy for carboplatin has been reported to be highly successful [10,11]; however, its administration is cumbersome and there are disadvantages in terms of temporal constraints. In addition, despite the use of an extensive desensitization regimen, two deaths have been reported [12]. Furthermore, administration of another platinum drug, nedaplatin $[9,13]$, is not currently recommended as the frequency of hypersensitivity reaction to nedaplatin is relatively high (27\%) [13]. In contrast, cisplatin administration for patients who developed carboplatin hypersensitivity reaction as alternative treatments have only been reported in a small number of patients, and most reports have mentioned only the short-term safety of cisplatin administration and not on its long-term efficacy $[14,15]$.

Since 2011, we have performed weekly administration of paclitaxel and cisplatin (wTP) in consecutive patients who developed carboplatin hypersensitivity reaction. Compared to dose-dense paclitaxel plus triweekly carboplatin, wTP with low-dose cisplatin has been reported to produce less hematologic toxicity, renal dysfunction, and anorexia, although the therapeutic effects of both regimens were similar [16]. The aim of this observational study was to investigate the safety and efficacy of wTP in patients with carboplatin hypersensitivity reaction.

\section{Materials and Methods}

\subsection{Patients and Study Design}

This was a retrospective, single-center study. The study was approved by the Institutional Review Board (\#3734) at Chiba University. We retrospectively enrolled a series of 86 consecutive patients who received wTP owing to carboplatin hypersensitivity reaction while undergoing paclitaxel carboplatin chemotherapy for ovarian, fallopian, and primary peritoneal cancer at our hospital from January 2011 to December 2019. Informed consent was obtained from all patients. Weekly paclitaxel and carboplatin were administered at initial treatment or at recurrent treatment to patients with platinum-sensitive disease during the study period. Patients who developed hypersensitivity reactions to carboplatin were administered wTP consecutively. Seventeen patients $(20 \%)$ received carboplatin with slow infusion rates after hypersensitivity reaction to carboplatin; however, they again developed hypersensitivity. Desensitization therapy for carboplatin was not applied since the study was performed at an outpatient chemotherapy unit. The patients' data included medical history, primary site, histology, stage, previous regimen, number of carboplatin cycles, severity of hypersensitivity reaction to carboplatin, number of wTP cycles, and presence and severity of hypersensitivity reaction to cisplatin. The primary endpoint was the incidence of patients who were able to receive wTP for at least 1 cycle throughout the 
study period. The secondary endpoint was treatment efficacy. Patients who completed wTP were defined as follows: (1) received more than six cycles of wTP without a hypersensitivity reaction or disease progression, (2) experienced a hypersensitivity reaction to cisplatin but the hypersensitivity reaction occurred after six cycles, and (3) received a total of six cycles of platinum chemotherapy together with the previous regimen (carboplatin). We also investigated the response rate of wTP, progression-free survival (PFS), and overall survival (OS).

Tumor response was evaluated based on the Response Evaluation Criteria in Solid Tumors, version 1.1. A complete response (CR) was defined as disappearance of all assessable target lesions without evidence of new lesions. Partial response (PR) was defined as at least $30 \%$ reduction in the sum of the longest diameter of all target lesions. Progressive disease (PD) was defined as at least a $20 \%$ increase in the sum of the longest diameter of all target lesions or development of new lesions. Stable disease (SD) was defined as any condition not meeting the aforementioned criteria.

\subsection{Protocol for wTP}

Similar to previous reports $[17,18]$, patients received paclitaxel $\left(80 \mathrm{mg} / \mathrm{m}^{2}\right)$ and cisplatin $\left(25 \mathrm{mg} / \mathrm{m}^{2}\right)$ on days 1,8 , and 15 of a 4-week period. If the patient's condition was good, the 1-week washout was omitted. Paclitaxel and cisplatin were dissolved separately in $250 \mathrm{cc}$ of saline, and paclitaxel was administered over $1 \mathrm{~h}$, followed by cisplatin over $1 \mathrm{~h}$. We instructed patients to drink $1 \mathrm{~L}$ of oral rehydration solution prior to treatment and administered no further infusion. Dexamethasone $(9.9 \mathrm{mg})$, d-chlorpheniramine maleate $(5 \mathrm{mg})$, and the histamine $\mathrm{H} 2$-receptor antagonist famotidine $(20 \mathrm{mg})$ were administered as premedication on the day. Full premedication on the previous day was not performed.

\subsection{Severity of Hypersensitivity Reaction}

The severity of the hypersensitivity reaction was graded according to the allergic reaction category of the Common Terminology Criteria for Adverse Events (CTCAE) ver. 4.0: Grade 1 (G1): transient flushing or rash, drug fever $<38^{\circ} \mathrm{C}$, intervention not indicated, Grade 2 (G2): intervention or infusion interruption indicated, responds promptly to symptomatic treatment, prophylactic medication indicated for $\leq 24 \mathrm{~h}$, Grade 3: prolonged, recurrence of symptoms following initial improvement, hospitalization indicated for clinical sequelae, Grade 4: life-threatening consequences, urgent intervention indicated, and Grade 5: death. The hypersensitivity reaction to carboplatin was confirmed when allergic reactions occurred during or at the end of carboplatin infusion.

\subsection{Statistical Analysis}

Pearson's chi-test was used for categorical variables. The Kaplan-Meier method was used to estimate the cumulative incidence of hypersensitivity reaction, the median PFS, and OS curves for each treatment arm. Comparisons between the intergroup were performed using two-sided log-rank and Wilcoxon square tests. PFS was defined as the interval between the administration of cisplatin and progressive disease or death. OS was defined as the interval between cisplatin administration and death. All data were analyzed based on the intention-to-treat principle. All tests were two-sided. A $p$-value of $<0.05$ was considered statistically significant. All analyses were performed using JMP software, version 11.0 (SAS, Cary, NC, USA).

\section{Results}

\subsection{Patients Characteristics}

Patient characteristics are shown in Table 1. The median age of the patients was 59 years. The primary site was the ovary in $53(62 \%)$ patients, the fallopian tube in $26(30 \%)$ patients, and the peritoneum in $7(8 \%)$ patients. Of the 86 patients, $71(83 \%)$ had advanced disease (stage III/IV) at the initial diagnosis. High-grade serous carcinoma was observed in $60(70 \%)$ patients, followed by clear cell carcinoma in $16(19 \%)$ patients. 
A total of 73 patients (85\%) developed a hypersensitivity reaction to carboplatin when receiving weekly paclitaxel and carboplatin \pm bevacizumab in the previous platinumbased chemotherapy. The median number of carboplatin cycles administered before the occurrence of hypersensitivity reaction was 8 (IQR: 6-11, range: 1-24). The hypersensitivity reactions to carboplatin were graded as G1, G2, and G3 in $57(66 \%), 26(30 \%)$, and $1(1 \%)$ patient, respectively. The number of patients who received wTP during the first, second, and third or more lines was $21(24 \%), 35(41 \%)$, and $30(35 \%)$ patients, respectively. A total of 35 patients ( $41 \%$ ) received wTP with bevacizumab.

Table 1. Patients characteristics.

\begin{tabular}{|c|c|c|}
\hline Variations & No. of Patients & Incidence (\%) \\
\hline Age (years) & \multirow{2}{*}{\multicolumn{2}{|c|}{$59(51-67)$}} \\
\hline Median, (IQR) & & \\
\hline \multicolumn{3}{|l|}{ Primary site } \\
\hline Ovary & 53 & 62 \\
\hline Peritoneum & 7 & 8 \\
\hline Fallopian Tube & 26 & 30 \\
\hline \multicolumn{3}{|l|}{ Primary FIGO stage } \\
\hline $\mathrm{I} / \mathrm{II}$ & 15 & 17 \\
\hline III/IV & 71 & 83 \\
\hline \multicolumn{3}{|l|}{ BRCA status } \\
\hline BRCA 1 mutation+ & 5 & 6 \\
\hline BRCA 2 mutation+ & 1 & 1 \\
\hline BRCA negative & 11 & 13 \\
\hline Unknown & 69 & 80 \\
\hline \multicolumn{3}{|l|}{ Histology } \\
\hline Serous carcioma, high-grade & 60 & 70 \\
\hline Endometrioid carcinoma & 4 & 5 \\
\hline Clear cell carcioma & 16 & 19 \\
\hline Others & 6 & 7 \\
\hline \multicolumn{3}{|l|}{ Previous platinum-based chemotherapy } \\
\hline Triweekly paclitaxel (docetaxel) and carboplatin \pm Bev & 13 & 15 \\
\hline Weekly paclitaxel and carboplatin \pm Bev & 73 & 85 \\
\hline \multicolumn{3}{|l|}{ No. of cycles in previous carboplatin } \\
\hline Median, $(\mathrm{IQR})$ & & \\
\hline $2-6$ & 28 & 33 \\
\hline $7-12$ & 42 & 49 \\
\hline $13-$ & 16 & 19 \\
\hline \multicolumn{3}{|l|}{ Grade of previous carboplatin hypersensitivity reaction } \\
\hline 1 & 57 & 66 \\
\hline 2 & 26 & 30 \\
\hline 3 & 1 & 1 \\
\hline unknown & 1 & 1 \\
\hline \multicolumn{3}{|l|}{ Timing of weekly paclitaxel and cisplatin } \\
\hline Initial treatment & 21 & 24 \\
\hline First recurrent treatment & 35 & 41 \\
\hline Second or more recurrent treatment & 30 & 35 \\
\hline
\end{tabular}

Abbreviations: Bev, bevacizumab; BRCA; breast cancer susceptibility, FIGO, International Federation of Gynecology and Obstetrics; IQR, interquartile range.

\subsection{Safety}

WTP was performed for a median of four cycles (IQR: 3-7), and 71 patients (83\%) were able to administer cisplatin for at least one cycle. Hypersensitivity reaction to cisplatin was observed in 15 patients (10 at G1 and 5 at G2). There were no deaths in all cohorts. The cumulative rate of hypersensitivity reaction to cisplatin was $17 \%$ (Figure 1). Seven (47\%) and 
eight (53\%) patients experienced hypersensitivity reactions to cisplatin within fewer than four and after five or more cycles of wTP, respectively. Two patients experienced hypersensitivity to cisplatin during the first cycle of wTP. In all 15 patients, the hypersensitivity reaction was ameliorated by steroid administration. There was no association between the occurrence of hypersensitivity reaction to cisplatin and the addition of bevacizumab (no bevacizumab: $6 / 51 ; 11.8 \%$ vs. bevacizumab: $9 / 35 ; 25.7 \%, p=0.094$ ).

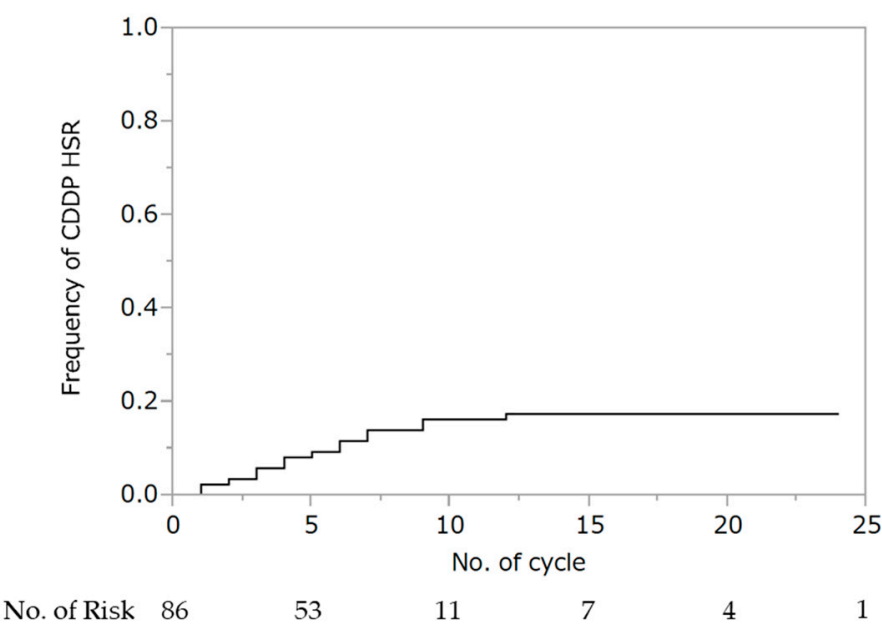

Figure 1. Cumulative rate of hypersensitivity reaction to cisplatin. Abbreviations: HSR, hypersensitivity reaction.

\subsection{Relationship between the Severity of Carboplatin Hypersensitivity and the Occurrence of Cisplatin Hypersensitivity}

The incidence of hypersensitivity reaction to cisplatin was $11 / 58(19 \%)$ in patients who experienced a G1 hypersensitivity reaction to carboplatin and $3 / 27(11 \%)$ in patients who experienced a G2 hypersensitivity reaction to carboplatin. One patient experienced unknown grade of hypersensitivity reaction to carboplatin. There was no association between the severity of the hypersensitivity reaction to carboplatin and the occurrence of hypersensitivity reaction to cisplatin $(p=0.363)$.

\subsection{Efficacy of Treatment}

A total of 55 patients (64\%) completed wTP treatment, as defined in the Methods Section: $9(10 \%)$ patients discontinued due to hypersensitivity reaction to cisplatin within 6 cycles, $1(1 \%)$ patient discontinued due to renal toxicity (grade 2 ) at the 6th cycle, and $21(24 \%)$ patients discontinued due to progressive disease within 6 cycles (Figure 2).

The response of 61 patients who had measurable disease was as follows: CR 14 (23\%), PR $16(26 \%)$, SD 13 (21\%), and PD in 18 patients (30\%). Notably, the response rate was $49 \%$. We could not evaluate the response of 25 patients who received one or two cycles of wTP after carboplatin hypersensitivity reaction or who received wTP during adjuvant chemotherapy after debulking surgery.

The median PFS and OS after the initiation of wTP was 10.6 months (95\% CI: 7.7-17.7) and 25.9 months (95\% CI: 19.0-50.2), respectively (Figure 3). For patients who developed a hypersensitivity reaction to carboplatin during first-line chemotherapy $(n=21$, stage III: 10 patients, stage IV: 11 patients), the median PFS was 24.4 months (95\% CI: 10.6-44.6) and OS was 50.2 months (95\% CI: 14.0-not reached), respectively (Figure 4). 


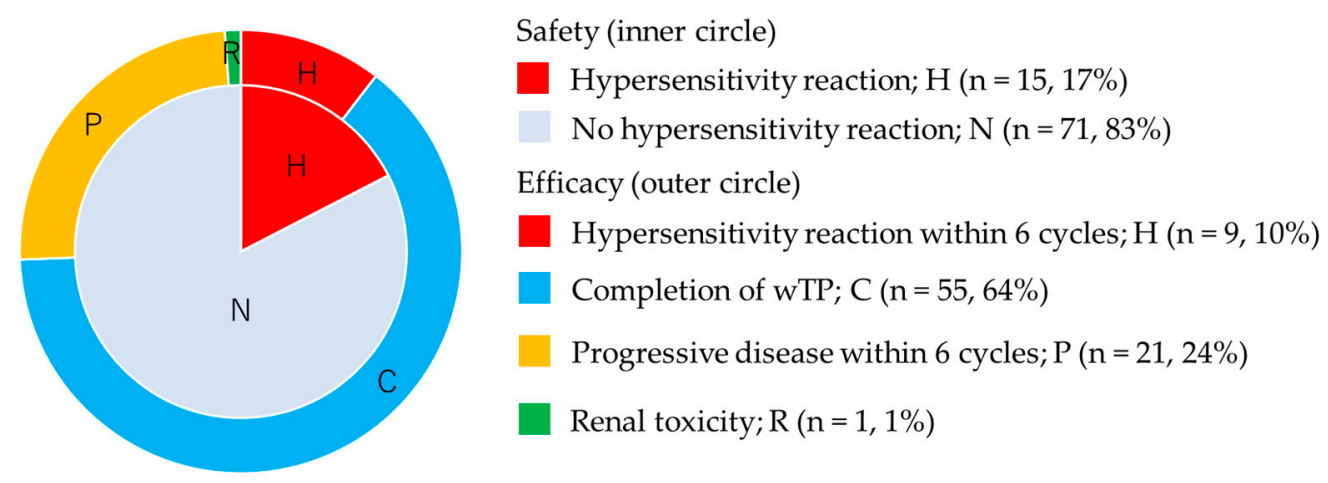

Figure 2. Safety and long-term efficacy of weekly paclitaxel and cisplatin. Inner circle shows safety and outer circle shows long-term efficacy.
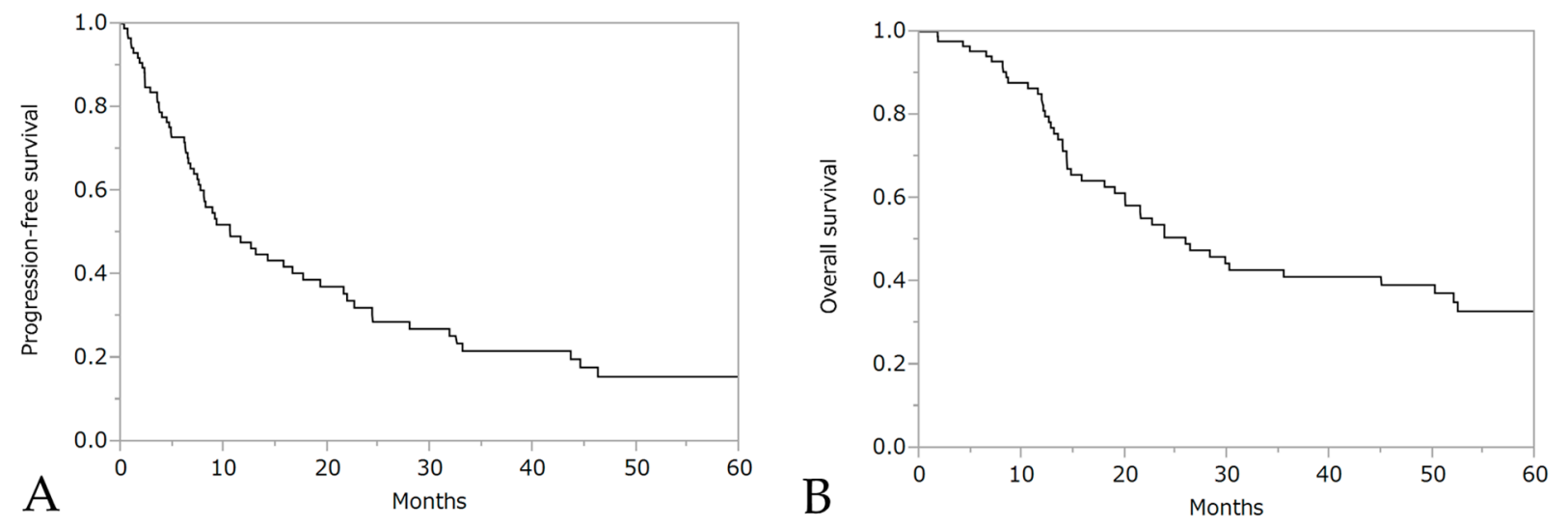

$\begin{array}{llllllllllllll}\text { No. of Risk } & 86 & 38 & 23 & 17 & 12 & 7 & 5 & \text { No. of Risk } & 86 & 67 & 42 & 29 & 23\end{array}$

Figure 3. Progression-free survival (PFS) (A) and overall survival (OS) (B) after the initiation of wTP.
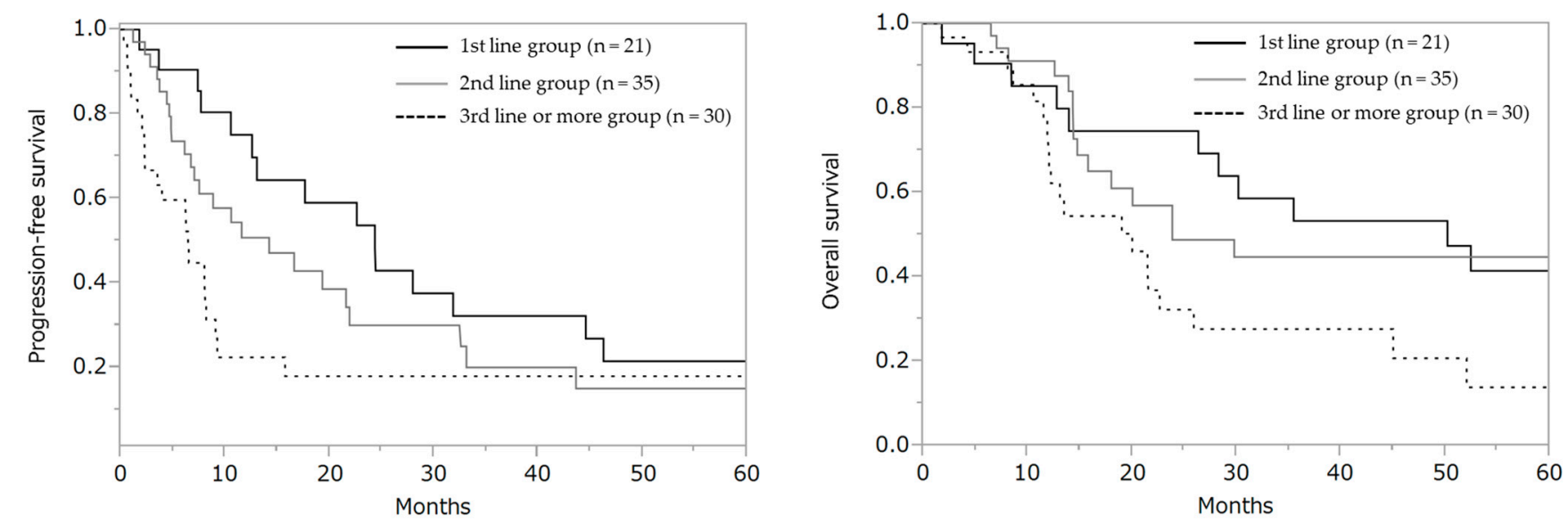

\begin{tabular}{|c|c|c|c|c|c|c|c|c|c|c|c|c|c|c|}
\hline No. of Risk & 21 & 16 & 12 & 8 & 7 & 4 & 2 & No. of Risk & 21 & 17 & 15 & 13 & 10 & 10 \\
\hline & 35 & 18 & 10 & 7 & 5 & 3 & 2 & & 35 & 29 & 16 & 12 & 10 & 9 \\
\hline & 30 & 6 & 3 & 3 & 2 & 2 & 1 & & 30 & 23 & 13 & 6 & 5 & 4 \\
\hline
\end{tabular}

Figure 4. Progression-free survival and overall survival according to each line. Progression-free survival (A) and overall survival (B) after wTP initiation.

To evaluate the treatment efficacy of wTP during initial treatment, we have compared the prognosis between 21 patients who received wTP with carboplatin hypersensitivity 
reaction and 334 patients who received weekly paclitaxel and carboplatin without carboplatin hypersensitivity reaction in the initial treatment at our institution. We show the patient characteristics in Table A1 and survival analyses in Figure A1 of the Appendix. There was no significant difference between the intergroups in PFS and OS. The treatment efficacy of wTP was not inferior to those of weekly paclitaxel and carboplatin in the initial treatment.

\section{Discussion}

\subsection{Key Findings of This Study}

This study investigated consecutive patients over an 8-year period who received identical regiments of wTP owing to the occurrence of hypersensitivity reaction to carboplatin. The safety and efficacy of cisplatin administration was confirmed in patients with hypersensitivity reactions to carboplatin. Among the studies reported to date, it is the most uniform and has the highest number of patients. In total, 86 patients who developed hypersensitivity reactions to carboplatin were treated with wTP, and $83 \%$ of the patients were able to continue the treatment without a hypersensitivity reaction to cisplatin There were no deaths due to hypersensitivity reaction to cisplatin. As shown in this study and reports to date, it is acceptable to administer cisplatin to patients who developed hypersensitivity reactions to carboplatin, and wTP can provide a clinical biomarker to show that patients receiving this treatment are platinum-sensitive [8]. This treatment strategy may serve as a bridge to PARP inhibitor maintenance therapy.

\subsection{Safety of $w T P$}

$83 \%$ of the patients received treatment without a hypersensitivity reaction to cisplatin, and there were no deaths due to the hypersensitivity reaction to cisplatin. In a previous report, Bergamini et al. reported that 5 of 38 patients (13.2\%) developed a hypersensitivity reaction to mild or moderate cisplatin [15]. Kolomeyevskaya et al. analyzed 19 patients who received cisplatin but observed no hypersensitivity reaction [19]. Callahan et al. also reported that 11 of 59 patients $(18.6 \%)$ who developed hypersensitivity reaction to carboplatin [14], including 24 patients in their experience and 35 patients in previous reports, experienced hypersensitivity reaction after the administration of cisplatin; of these 59 patients, 2 died [20,21]. Combining their data [14,15,19] with our report, the incidence of the hypersensitivity reaction to cisplatin in patients with hypersensitivity reaction to carboplatin was 31/202 (15.3\%), with two deaths (1.0\%). In addition, there was no association between the severity of the hypersensitivity reaction to carboplatin and the incidence of hypersensitivity reaction to cisplatin in our study. Although both carboplatin and cisplatin are platinum agents, their cross-reactivity is reported to be low [22].

\subsection{Efficacy of wTP}

In terms of long-term efficacy, the majority of patients (54 patients, $63 \%$ ) completed the scheduled wTP, including 6 patients who experienced hypersensitivity reaction to cisplatin after 6 cycles. Only 9 patients $(10 \%)$ discontinued treatment within 6 cycles due to hypersensitivity reaction to cisplatin. In terms of the use of PARP inhibitors [8], patients who received more than 4 cycles of wTP and had complete or partial responses would be able to receive the benefits of a PARP inhibitor.

\subsection{Weekly versus Triweekly Carboplatin in Hypersensitivity Reaction}

Compared with previous studies, a large sample size was obtained despite this being a single-institution study. One of the reasons may be that we chose weekly paclitaxelcarboplatin chemotherapy as the first-line chemotherapy, which resulted in a higher incidence of hypersensitivity reaction to carboplatin than triweekly administration. Reportedly, the incidence of hypersensitivity reactions to carboplatin is higher in weekly treatment for low-grade glioma in children $[23,24]$. A higher incidence of hypersensitivity reaction has been reported in weekly paclitaxel-carboplatin chemotherapy when used as a primary 
treatment for advanced ovarian cancer $[25,26]$. The International Collaboration on Ovarian Neoplasms (ICON)-8 study [27] revealed that allergic reactions (of any grade) were significantly higher in the group of patients administered weekly carboplatin compared with triweekly carboplatin (triweekly carboplatin: 110/911 patients, vs. weekly carboplatin: 90/420 patients; $p<0.001)$.

\subsection{Advantages of Weekly Paclitaxel and Cisplatin Chemotherapy}

We chose weekly administration of paclitaxel and cisplatin because the use of weekly cisplatin reduces gastrointestinal symptoms, such as nausea, vomiting, and renal toxicity. A previous phase III study reported that gastrointestinal symptoms and renal toxicity were higher in triweekly cisplatin than triweekly carboplatin [28,29]. Moreover, dose-dependent administration of paclitaxel has been shown to be effective in Japan [30]. Reportedly, this regimen has mild renal toxicity and hematologic toxicity, even in other cancers $[17,18]$. Our historical cohort analysis revealed that the treatment efficacy of wTP was not inferior to those of weekly paclitaxel and carboplatin in the initial treatment. The administration of cisplatin may be useful in terms of safety and efficacy for patients with hypersensitivity reactions to carboplatin in first-line chemotherapy treatment.

\subsection{Strengths and Limitation}

The strengths of this study include a large and homogenous cohort of all 86 patients who received wTP for a platinum-sensitive disease. The limitation was that the use of a weekly schedule of platinum is uncommon, at least in Europe and the USA, along with the retrospective design.

\section{Conclusions}

The administration of cisplatin to patients with hypersensitivity reaction to carboplatin was a concern owing to two deaths reported previously $[20,21]$. However, when combining the results of our study and previous reports $[14,15,19]$, the incidence of hypersensitivity reaction to cisplatin was shown to be $31 / 183(16.9 \%)$, with two deaths $[16,17](1.1 \%)$. While desensitization therapy is cumbersome and there are disadvantages in terms of temporal constraints, wTP is simple and easy to administer. It is acceptable to administer cisplatin to patients who develop a hypersensitivity reaction to carboplatin. Although the use of a weekly schedule of platinum is uncommon, at least in Europe and the USA, this treatment strategy may serve as a bridge to PARP inhibitor maintenance therapy.

Author Contributions: Conceptualization, S.T. and M.S.; methodology, S.T.; formal analysis, S.T.; investigation, S.T.; data curation, S.T. and K.N.; writing-original draft preparation, S.T.; writingreview and editing, S.T., K.N., A.M. and S.O.; visualization, S.T.; supervision, M.S.; project administration, S.T. All authors have read and agreed to the published version of the manuscript.

Funding: This research received no external funding.

Institutional Review Board Statement: The study was conducted according to the guidelines of the Declaration of Helsinki, and approved by the Institutional Review Board at Chiba University on 30 June 2020 (protocol code \#3734).

Informed Consent Statement: Patient consent was waived due to the retrospective design of this study. An opt-out system was used instead.

Conflicts of Interest: The authors declare no conflict of interest. 


\section{Appendix A. Comparison of Survival between Patients with Carboplatin Hypersensitivity Reaction or Not during the Initial Treatment}

Table A1. Comparison of characteristics between patients with carboplatin hypersensitivity reaction or not.

\begin{tabular}{|c|c|c|c|c|c|}
\hline \multirow{2}{*}{$\begin{array}{c}\text { Patient Characteristics } \\
\text { (Variable) }\end{array}$} & \multicolumn{2}{|c|}{ CBDCA HSR + $(n=21)$} & \multicolumn{2}{|c|}{ CBDCA HSR $-(n=334)$} & \multirow{2}{*}{$p$-Value } \\
\hline & No & $\%$ & No & $\%$ & \\
\hline Age (median, years, IQR) & \multicolumn{2}{|c|}{$63(51-71)$} & \multicolumn{2}{|c|}{$62(51-70)$} & 0.86 \\
\hline \multicolumn{6}{|l|}{ FIGO stage } \\
\hline III & 10 & $(48 \%)$ & 212 & $(63 \%)$ & \\
\hline IV & 11 & $(52 \%)$ & 122 & $(37 \%)$ & 0.152 \\
\hline \multicolumn{6}{|l|}{ Performance status } \\
\hline $0-1$ & 6 & $(29 \%)$ & 232 & $(69 \%)$ & \\
\hline $2-4$ & 15 & $(71 \%)$ & 102 & $(31 \%)$ & $<0.001$ \\
\hline \multicolumn{6}{|l|}{ Primary site } \\
\hline Ovary & 10 & $(48 \%)$ & 205 & $(61 \%)$ & \\
\hline Fallopian tube & 10 & $(48 \%)$ & 101 & $(30 \%)$ & 0.103 \\
\hline Peritoneum & 1 & $(5 \%)$ & 28 & $(8 \%)$ & \\
\hline \multicolumn{6}{|l|}{ Histology } \\
\hline Serous carcinoma, high grade & 16 & $(76 \%)$ & 232 & $(69 \%)$ & \\
\hline Clear cell carcinoma & 1 & $(5 \%)$ & 37 & $(11 \%)$ & \\
\hline Endometrioid carcinoma & 0 & $(0 \%)$ & 27 & $(8 \%)$ & 0.309 \\
\hline Mucinous carcinoma & 0 & $(0 \%)$ & 5 & $(1 \%)$ & \\
\hline Others & 4 & $(19 \%)$ & 33 & $(10 \%)$ & \\
\hline \multicolumn{6}{|l|}{ CA125 } \\
\hline Median (IQR), IU/mL & \multicolumn{2}{|c|}{$1357(567-2719)$} & \multicolumn{2}{|c|}{764 (303-1987) } & 0.067 \\
\hline \multicolumn{6}{|l|}{ PCI } \\
\hline Median (IQR) & \multicolumn{2}{|c|}{$19(14-22)$} & \multicolumn{2}{|c|}{$14(5.5-20)$} & 0.045 \\
\hline \multicolumn{6}{|l|}{ Treatment } \\
\hline Primary surgery & 3 & $(14 \%)$ & 149 & $(45 \%)$ & \\
\hline Interval surgery & 15 & $(71 \%)$ & 162 & $(49 \%)$ & 0.258 \\
\hline Chemotherapy only & 3 & $(14 \%)$ & 23 & $(7 \%)$ & \\
\hline \multicolumn{6}{|l|}{ Residual disease } \\
\hline No residual tumor & 18 & $(86 \%)$ & 278 & $(83 \%)$ & \\
\hline$\leq 10 \mathrm{~mm}$ & 0 & $(0 \%)$ & 24 & $(7 \%)$ & 0.374 \\
\hline$>10 \mathrm{~mm}$ & 3 & $(14 \%)$ & 32 & $(10 \%)$ & \\
\hline
\end{tabular}

No significant differences were seen in patient characteristics, except performance status between patients with carboplatin hypersensitivity reaction or not.

A

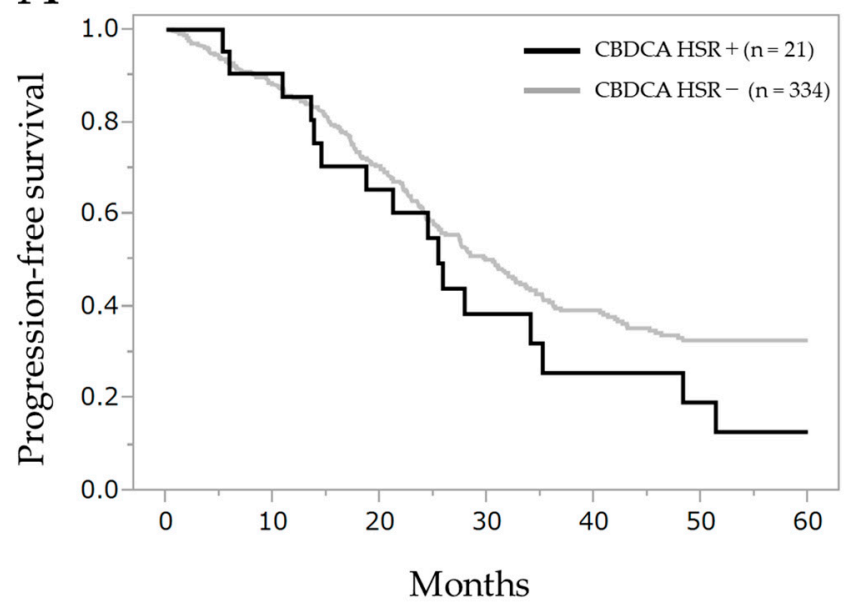

B

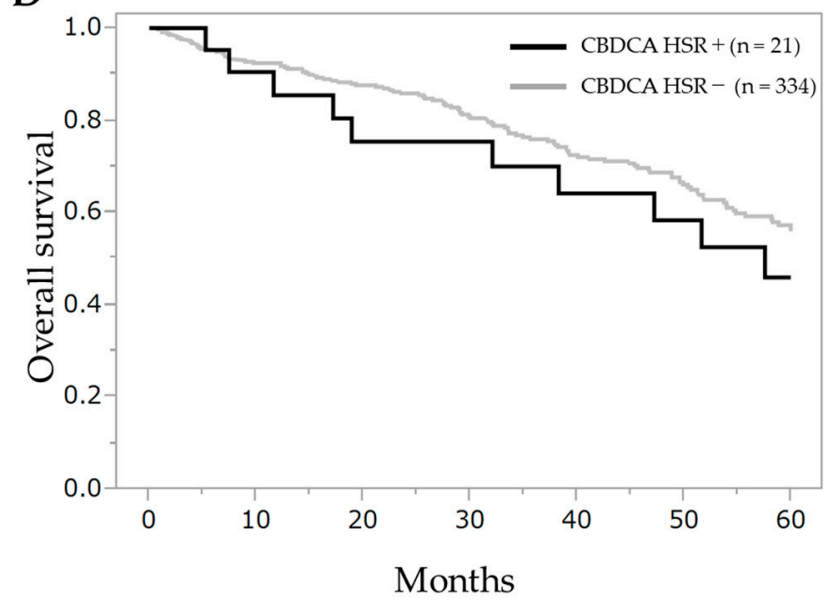

Figure A1. Comparison of progression-free survival and overall survival between patients with carboplatin hypersensitivity reaction or not. (A) Progression-free survival and (B) overall survival after the initial treatment. CBDCA HSR: carboplatin hypersensitivity reaction. 
No significant differences were observed in the intergroup corresponding to progressionfree survival (log-rank test, $p=0.196$, Wilcoxon test, $p=0.435$ ) and overall survival (log-rank test, $p=0.344$, Wilcoxon test, $p=0.331$ ). Progression-free survival in patients with carboplatin hypersensitivity reaction or not was 25.4 months (95\% CI 14.5-35.2) and 30.5 months (95\% CI 26.1-33.7), respectively. The overall survival was 57.6 months (95\% CI 32.1-110.2) and 80.0 months (95\% CI 64.9-101.6), respectively. Median follow-up period was 39.4 months.

\section{References}

1. Aghajanian, C.; Blank, S.V.; Goff, B.A.; Judson, P.L.; Teneriello, M.G.; Husain, A.; Sovak, M.A.; Yi, J.; Nycum, L.R. OCEANS: A Randomized, Double-Blind, Placebo-Controlled Phase III Trial of Chemotherapy with or without Bevacizumab in Patients with Platinum-Sensitive Recurrent Epithelial Ovarian, Primary Peritoneal, or Fallopian Tube Cancer. J. Clin. Oncol. 2012, 30, $2039-2045$. [CrossRef] [PubMed]

2. Ledermann, J.; Harter, P.; Gourley, C.; Friedlander, M.; Vergote, I.; Rustin, G.; Scott, C.; Meier, W.; Shapira-Frommer, R.; Safra, T.; et al. Olaparib Maintenance Therapy in Platinum-Sensitive Relapsed Ovarian Cancer. N. Engl. J. Med. 2012, 366, 1382-1392. [CrossRef]

3. Bartoletti, M.; Pelizzari, G.; Gerratana, L.; Bortot, L.; Lombardi, D.; Nicoloso, M.S.; Scalone, S.; Giorda, G.; Baldassarre, G.; Sorio, R.; et al. Bevacizumab or PARP-Inhibitors Maintenance Therapy for Platinum-Sensitive Recurrent Ovarian Cancer: A Network Meta-Analysis. Int. J. Mol. Sci. 2020, 21, 3805. [CrossRef]

4. Pfisterer, J.; Shannon, C.M.; Baumann, K.; Rau, J.; Harter, P.; Joly, F.; Sehouli, J.; Canzler, U.; Schmalfeldt, B.; Dean, A.P.; et al. Bevacizumab and platinum-based combinations for recurrent ovarian cancer: A randomised, open-label, phase 3 trial. Lancet Oncol. 2020, 21, 699-709. [CrossRef]

5. Meeting Library I Randomized Phase III Study to Evaluate the Impact of Secondary Cytoreductive Surgery in Recurrent Ovarian Cancer: Final Analysis of AGO DESKTOP III/ENGOT-ov20. Available online: https:/ / meetinglibrary.asco.org/record/185438 /abstract (accessed on 29 May 2020).

6. Coleman, R.L.; Spirtos, N.M.; Enserro, D.; Herzog, T.J.; Sabbatini, P.; Armstrong, D.K.; Kim, J.-W.; Park, S.-Y.; Kim, B.-G.; Nam, J.-H.; et al. Secondary Surgical Cytoreduction for Recurrent Ovarian Cancer. N. Engl. J. Med. 2019, 381, 1929-1939. [CrossRef] [PubMed]

7. Markman, M.; Kennedy, A.; Webster, K.; Elson, P.; Peterson, G.; Kulp, B.; Belinson, J. Clinical Features of Hypersensitivity Reactions to Carboplatin. J. Clin. Oncol. 1999, 17, 1141. [CrossRef] [PubMed]

8. Morgan, R.D.; Clamp, A.R.; Evans, D.G.R.; Edmondson, R.J.; Jayson, G.C. PARP inhibitors in platinum-sensitive high-grade serous ovarian cancer. Cancer Chemother. Pharmacol. 2018, 81, 647-658. [CrossRef] [PubMed]

9. Michikami, H.; Minaguchi, T.; Ochi, H.; Onuki, M.; Okada, S.; Matsumoto, K.; Satoh, T.; Oki, A.; Yoshikawa, H. Safety and efficacy of substituting nedaplatin after carboplatin hypersensitivity reactions in gynecologic malignancies. J. Obstet. Gynaecol. Res. 2012, 39, 330-335. [CrossRef] [PubMed]

10. Lee, C.-W.; Matulonis, U.A.; Castells, M. Rapid inpatient/outpatient desensitization for chemotherapy hypersensitivity: Standard protocol effective in 57 patients for 255 courses. Gynecol. Oncol. 2005, 99, 393-399. [CrossRef]

11. Vetter, M.H.; Khan, A.; Backes, F.J.; Bixel, K.; Cohn, D.E.; Copeland, L.J.; Fowler, J.M.; Salani, R.; Li, Q.; O'Malley, D.M. Outpatient de-sensitization of patients with moderate (high-risk) to severe platinum hypersensitivity reactions. Gynecol. Oncol. 2019, 152, 316-321. [CrossRef]

12. Markman, M. Hypersensitivity Reactions to Carboplatin. Gynecol. Oncol. 2002, 84, 353-354. [CrossRef]

13. Arimoto, T.; Oda, K.; Nakagawa, S.; Kawana, K.; Tsukazaki, T.; Adachi, K.; Matsumoto, Y.; Yano, T.; Kozuma, S.; Taketani, Y. Re-treatment with nedaplatin in patients with recurrent gynecological cancer after the development of hypersensitivity reac-tion to carboplatin. J. Obstet. Gynaecol. Res. 2013, 39, 336-340. [CrossRef]

14. Callahan, M.B.; Lachance, J.A.; Stone, R.L.; Kelsey, J.; Rice, L.W.; Jazaeri, A.A. Use of cisplatin without desensitization after car-boplatin hypersensitivity reaction in epithelial ovarian and primary peritoneal cancer. Am. J. Obstet. Gynecol. 2007, 197, 199.e1-199.e5. [CrossRef]

15. Bergamini, A.; Pisano, C.; Di Napoli, M.; Arenare, L.; Della Pepa, C.; Tambaro, R.; Facchini, G.; Gargiulo, P.; Rossetti, S.; Mangili, G.; et al. Cisplatin can be safely administered to ovarian cancer patients with hypersensitivity to carboplatin. Gynecol. Oncol. 2017, 144, 72-76. [CrossRef]

16. Huang, C.-Y.; Cheng, M.; Lee, N.-R.; Huang, H.-Y.; Lee, W.-L.; Chang, W.-H.; Wang, P.-H. Comparing Paclitaxel-Carboplatin with Paclitaxel-Cisplatin as the Front-Line Chemotherapy for Patients with FIGO IIIC serous-Type Tubo-Ovarian Cancer. Int. J. Environ. Res. Public Health 2020, 17, 2213. [CrossRef] [PubMed]

17. Nagata, N.; Kimura, M.; Hirabayashi, N.; Tuburaya, A.; Murata, T.; Kondo, K.; Fukuda, Y.; Kobayashi, M.; Miyashita, Y.; Nakao, A.; et al. Phase II study of weekly paclitaxel and cisplatin combination therapy for advanced or recurrent gastric cancer. Hepatogastroenterology 2008, 55, 1846-1850. 
18. Tsuburaya, A.; Nagata, N.; Cho, H.; Hirabayashi, N.; Kobayashi, M.; Kojima, H.; Munakata, Y.; Fukushima, R.; Kameda, Y.; Shimoda, T.; et al. Phase II trial of paclitaxel and cisplatin as neoadjuvant chemotherapy for locally advanced gastric cancer. Cancer Chemother Pharmacol. 2013, 71, 1309-1314. [CrossRef] [PubMed]

19. Kolomeyevskaya, N.V.; Lele, S.B.; Miller, A.; Riebandt, G.C.; Blum, B.L.; Odunsi, K.O.; Frederick, P.J. Oxaliplatin is a safe alternative option for patients with recurrent gynecologic cancers after hypersensitivity reaction to Carboplatin. Int. J. Gynecol. Cancer 2015, 25, 42-48. [CrossRef] [PubMed]

20. Zweizig, S.; Roman, L.D.; Muderspach, L.I. Death from Anaphylaxis to Cisplatin: A Case Report. Gynecol. Oncol. 1994, 53, 121-122. [CrossRef] [PubMed]

21. Dizon, D.S.; Sabbatini, P.J.; Aghajanian, C.; Hensley, M.L.; Spriggs, D.R. Analysis of Patients with Epithelial Ovarian Cancer or Fallopian Tube Carcinoma Retreated with Cisplatin after the Development of a Carboplatin Allergy. Gynecol. Oncol. 2002, 84, 378-382. [CrossRef]

22. Pasteur, J.; Favier, L.; Pernot, C.; Guerriaud, M.; Bernigaud, C.; Lepage, C.; Jouve, J.-L.; Isambert, N.; Collet, E. Low CrossReactivity Between Cisplatin and Other Platinum Salts. J. Allergy Clin. Immunol. Pract. 2019, 7, 1894-1900. [CrossRef]

23. Genc, D.B.; Canpolat, C.; Berrak, S.G. Clinical features and management of carboplatin-related hypersensitivity reactions in pediatric low-grade glioma. Support. Care Cancer 2011, 20, 385-393. [CrossRef]

24. Lafay-Cousin, L.; Sung, L.; Carret, A.S.; Hukin, J.; Wilson, B.; Johnston, D.L.; Zelcer, S.; Silva, M.; Odame, I.; Mpofu, C.; et al. Carboplatin hypersensitivity reaction in pediatric patients with low-grade glioma: A Canadian Pediatric Brain Tumor Consortium experience. Cancer 2008, 112, 892-899. [CrossRef]

25. Safra, T.; Menczer, J.; Bernstein, R.M.; Shpigel, S.; Matcejevsky, D.; Inbar, M.J.; Golan, A.; Grisaru, D.; Levy, T. Combined weekly carboplatin and paclitaxel as primary treatment of advanced epithelial ovarian carcinoma. Gynecol. Oncol. 2009, 114, 215-218. [CrossRef]

26. Pignata, S.; Scambia, G.; Katsaros, D.; Gallo, C.; Pujade-Lauraine, E.; De Placido, S.; Bologna, A.; Weber, B.; Raspagliesi, F.; Panici, P.B.; et al. Carboplatin plus paclitaxel once a week versus every 3 weeks in patients with advanced ovarian cancer (MITO-7): A randomised, multicentre, open-label, phase 3 trial. Lancet Oncol. 2014, 15, 396-405. [CrossRef]

27. Clamp, A.R.; James, E.C.; McNeish, I.A.; Dean, A.; Kim, J.-W.; O’Donnell, D.M.; Hook, J.; Coyle, C.; Blagden, S.; Brenton, J.D.; et al. Weekly dose-dense chemotherapy in first-line epithelial ovarian, fallopian tube, or primary peritoneal carcinoma treatment (ICON8): Primary progression free survival analysis results from a GCIG phase 3 randomised controlled trial. Lancet 2019, 394, 2084-2095. [CrossRef]

28. Ozols, R.F.; Bundy, B.N.; Greer, B.E.; Fowler, J.M.; Clarke-Pearson, D.; Burger, R.A.; Mannel, R.S.; DeGeest, K.; Hartenbach, E.M.; Baergen, R. Phase III Trial of Carboplatin and Paclitaxel Compared With Cisplatin and Paclitaxel in Patients With Optimally Resected Stage III Ovarian Cancer: A Gynecologic Oncology Group Study. J. Clin. Oncol. 2003, 21, 3194-3200. [CrossRef]

29. Du Bois, A.; Luck, H.J.; Meier, W.; Adams, H.P.; Mobus, V.; Costa, S.; Bauknecht, T.; Richter, B.; Warm, M.; Schroder, W.; et al. A randomized clinical trial of cisplatin/paclitaxel versus carboplatin/paclitaxel as first-line treatment of ovarian cancer. J. Natl. Cancer Inst. 2003, 95, 1320-1329. [CrossRef]

30. Katsumata, N.; Yasuda, M.; Takahashi, F.; Isonishi, S.; Jobo, T.; Aoki, D.; Tsuda, H.; Sugiyama, T.; Kodama, S.; Kimura, E.; et al. Dose-dense paclitaxel once a week in combination with carboplatin every 3 weeks for advanced ovarian cancer: A phase 3, open-label, randomised controlled trial. Lancet 2009, 374, 1331-1338. [CrossRef] 